\title{
High-frequency backscatter analysis of human articular cartilage
}

\author{
Nils Männicke ${ }^{1}$, Martin Schöne ${ }^{1}$, Mathias Gottwald ${ }^{2}$, Felix Göbel $^{3}$, Michael Oelze $^{4}$, Kay Raum ${ }^{1}$ \\ ${ }^{1}$ Julius-Wolff Institute, Charité Universitätsmedizin Berlin, Berlin, Germany \\ ${ }^{2}$ Department of Surgery, Hospital Köln-Holweide, Cologne, Germany \\ ${ }^{3}$ Department of Orthopaedics and Traumatology, Carl-von-Basedow-Klinikum, Merseburg, Germany \\ ${ }^{4}$ Department of Electrical Engineering, University of Illinois at Urbana-Champaign, Urbana, IL,USA
}

Email: nils.maennicke@charite.de

\begin{abstract}
-46 punch biopsies of human cartilage were collected and scanned in time-resolved $\mathrm{C}$-scan mode using a scanning acoustic microscope with a single-element $40-\mathrm{MHz}$ transducer. From these data, spectra were estimated from short time gates at regions with low inclination and averaged with respect to their time delay to the tissue surface to account for the curved shape of articular cartilage. The depth-dependent spectral slope and amplitude profiles were used to estimate the maximum values and the depth-dependent slope. The surface reflection was excluded from analysis. In addition, the homodyned $k$ distribution was used to model the signal amplitude of the envelope at tissue depths between 200 and $600 \mu \mathrm{m}$. The $k$ and $\mu$ parameters were estimated from the envelope data. KruskalWallis analyses of variance were carried out for the acoustic parameters with respect to subscores of the Mankin grading system that are related to cellular and structural abnormalities of the cartilage matrix. The grading was performed on 2D histological cross sections. Additionally the cell number density was assessed.

Moderate positive correlations between cell number and backscatter amplitude $\left(R^{2}=0.43\right)$ were observed. The backscatter amplitude parameters as well as $k$ were not statistically significantly different with respect to degenerative changes, whereas $\mu$ was significantly lower for advanced stages of degeneration. The spectral slope and the extrapolation to the surface of the spectral slope were significantly higher for samples without signs of degeneration. The highest significance levels and chi $^{2}$ values were obtained for the maximum spectral slope value within the superficial zone of cartilage.

The results suggest that amplitude based parameters alone are insufficient to discriminate different stages of spontaneous degeneration and that in contrast, spectral slope as well as envelope statistics are sensitive to osteoarthritic changes. Due to the correlation of backscatter amplitude and cell number, differences of the spectral slopes are likely due to cellular degenerations.
\end{abstract}

Keywords- backscatter, cartilage, degeneration, envelope statistics, Spectral analysis

\section{INTRODUCTION}

Only a few studies have investigated acoustic backscatter originating from the cartilage matrix for detection and characterization of cartilage degeneration and so far only integrated spectral amplitudes of the received signals have been considered, i.e. the apparent integrated backscatter $(A I B)$.
However, until now the origin of acoustic backscatter from cartilage tissue has not been fully identified.

Much of ultrasound backscatter arises from sub-wavelength structures. The spectrum of backscattered signal received by an ultrasound probe from a scattering tissue is determined by distribution, geometry, and acoustic impedance mismatch between scatterers and the surrounding medium. Theoretical formulations [1] and experimental applications [2] have been conducted to successfully utilize quantitative backscatter parameter for tissue characterization. However, for cartilage tissue the incorporation of these spectral features has not been achieved. One reason is that the estimation of spectra requires locally homogeneous scattering properties in the direction of sound propagation with axial dimensions on the order of several pulse lengths. The layered cartilage structure consists of three layers, in which cells and collagen fibrils gradually change geometry, density, and orientation, resulting in gradual changes of acoustic backscatter and bulk properties, e.g. speed of sound and attenuation [3].

To overcome these limitations, we applied threedimensional high-frequency ultrasound in combination with depth-dependent spectral analysis using short time gates to the analysis of healthy and degenerated human cartilage samples. The aim of this study was to analyze the diagnostic value of the frequency dependence and envelope statistics of backscattered signals.

\section{MATERIALS AND METHODS}

\section{A. Samples}

This work was based on the same set of measurements used in a previous study and reported in detail therein [4]. One to four punch biopsies (diameter: $8 \mathrm{~mm}$ ) of degenerated cartilage were obtained from the femoral condyles of 19 patients, yielding 38 samples with variable degrees of degeneration. Additionally, punch biopsies $(\mathrm{N}=10)$ from the femoral joint were obtained from four human cadavers with no degenerative joint disease. Furthermore, a sample containing finely powdered graphite particles immersed in agar was used as a reference material that mimics variations of the axial pressure amplitude in a scattering medium. 


\section{B. Histology}

Histological analysis was performed on demineralized and paraffin-embedded sections of the respective punch biopsies using routine histology processing and staining (Safranin-O, $\mathrm{HE})$. Cartilage degeneration was graded using the individual scoring categories of the 14-point Mankin-score [5]. Of particular interest were the structure score (referred to as Mankin I) and the scoring of cellular abnormalities (Mankin II). Moreover, the cells were counted in one histological section in a $1 \mathrm{~mm}^{2}$ square area directly below the cartilage surface.

\section{Ultrasound measurements}

Cartilage samples were immersed at $25^{\circ} \mathrm{C}$ in $\mathrm{PBS}$ and measured in C-scan mode (scan increment: $20 \mu \mathrm{m}$ in $x$ and $y$ direction) using the custom scanning acoustic microscope SAM200Ex $[4,6]$. The SAM is controlled by custom software (SAMEx, Q-BAM, Halle, Germany) and consists of a 3-axis high precision scanning stage, a $200 \mathrm{MHz}$ pulser/receiver (Panametrics 5900PR, Waltham, USA) and a $500 \mathrm{MSs}-1 \mathrm{~A} / \mathrm{D}-$ card (Gage CS8500, Gage Applied Technologies Inc., Lachine, Canada). A spherically-focused $40-\mathrm{MHz}$ transducer (f-number: 2.66, diameter: $3 \mathrm{~mm}$, NIH Resource Center for Ultrasound Transducer Technology, Los Angeles, CA, USA) was used, providing lateral and axial resolutions of $120 \mu \mathrm{m}$ and $50 \mu \mathrm{m}$, respectively. The center frequency at the focus was $37.5 \mathrm{MHz}$ and the $-6 \mathrm{~dB}$ range was $25-50 \mathrm{MHz}$. The pulse length (PL) was $60 \mathrm{~ns}$, the focal distance was $9 \mathrm{~mm}$ and the transducersample distance was adjusted such that the focal plane of the transducer was approximately $0.5 \mathrm{~mm}$ underneath the cartilage surface.

\section{Data Analysis}

\section{1) Local surface position and inclination}

For every scan point, the sample surfaces were approximated using a threshold method combined with spatial filtering, resulting in a map denoted by $d(x, y)$. The local inclination was estimated based on these surface positions [4]. The speed of sound of the coupling medium used for the conversion from pulse-echo travel times to spatial distances was $\mathrm{c}_{0}=1540 \mathrm{~m} / \mathrm{s}$. In this study, the region of interest included waveforms for which the inclination was between $0^{\circ}$ and $5^{\circ}$.

\section{2) Spectral analysis of backscatter}

The backscatter power spectra $|S(f, x, y, z)|^{2}$ at the lateral position $(x, y)$ and transducer gate distance $z$ were estimated by calculating the magnitude squared of the Fast Fourier Transform (FFT) of Hamming-windowed time signals with a duration of 2.5 PL.

\section{a) Reference spectrum}

One calibration spectrum $\left|S_{\text {ref }}\left(f, z_{0}\right)\right|^{2}$ obtained from the planar reflection of a polymethylmethacrylate (PMMA) block at the focus $z_{0}$ was used to compensate for the effects of system and transducer transfer functions. Amplitude correction for defocused positions was achieved by using the backscatter intensity from the agar-graphite phantom. The reference spectra of different axial positions can then be expressed as

$$
S_{\text {ref }}(f, z)=\frac{S_{\text {ref }}\left(f, z_{0}\right)}{R_{P M M A}} \cdot \frac{I_{\text {graphite }}(z)}{I_{\text {graphite }}\left(z_{0}\right)},
$$

whereas $R_{P M M A}=0.281$ is the reflection coefficient of PMMA and $I_{\text {graphite }}(z) / I_{\text {graphite }}\left(z_{0}\right)$ denotes the normalized depthdependent backscatter intensity from the graphite phantom within the $-6-\mathrm{dB}$ bandwidth of the transducer.

\section{b) Backscatter amplitude}

The apparent integrated backscatter $(A I B)$ quantifies the backscattered energy in the $-6-\mathrm{dB}$ bandwidth of the transducer from signals at different time gates. $A I B$ is a function of tissue depth $z_{s}$ relative to the cartilage surface $d(x, y)$ :

$$
\begin{gathered}
A I B\left(z_{s}\right)=\frac{1}{N_{x, y} \cdot \Delta f} \sum_{x, y}^{N_{x, y}} \sum_{\Delta f} 10 \cdot \log \left\langle\frac{|S(f, x, y, z)|^{2}}{\left|S_{r e f}(f, z)\right|^{2}}\right\rangle, \\
\text { with } \\
z=z_{s}+d(x, y) .
\end{gathered}
$$

The conversion from surface time delays to tissue depths was performed assuming a constant speed of sound of $c_{l}=1620$ $\mathrm{m} / \mathrm{s}$ within the cartilage tissue [3]. $N_{x, y}$ is the number of averaged waveforms.

\section{c) Backscatter frequency dependence}

Similar to the formulation of the $A I B$ in Eq. (2), difference spectra were calculated as a function of tissue depth. The difference spectra $W\left(f, z_{s}\right)$ were calculated as:

$$
\begin{aligned}
W\left(f, z_{s}\right) & =\left(\frac{1}{N_{x, y}} \sum_{x, y}^{N_{x, y}} 10 \cdot \log _{10}\left\langle|S(f, x, y, z)|^{2}\right\rangle\right)- \\
& 10 \cdot \log _{10}\left\langle\left|S_{r e f}\left(f, z_{0}\right)\right|^{2}\right\rangle \cdot
\end{aligned}
$$

Note that no amplitude correction was necessary since only relative differences of the spectra were targeted. Linear regression analysis was applied within the bandwidth of the transducer and the spectral slope was derived for each depth $z_{s}$. Because the $A I B$ has been well-established in assessing cartilage properties with ultrasound, we accordingly define this parameter as Apparent Frequency-dependence of Backscatter $(A F B)$.

\section{3) Depth-dependent backscatter parameters}

$A I B$ and $A F B$ were calculated as a function of depth using a sliding window technique [6]. The first window was centered at the detected surface position and the maximum depth was set to $2.5 \mathrm{~mm}$. For a strict differentiation between backscatter and the previously reported surface analysis, the surface reflection of the acquired voltage signal was gated out by adding the inverted signal multiplied with a Tukey window $(\alpha=0.5)$ at the center position of $d(x, y)$ with a gate length of 2.5 PL (Fig. 3b).

From each depth-dependent profile of $A I B$ and $A F B$ (Fig. $3 \mathrm{~d}, \mathrm{f})$, the following parameters were extracted. $A I B_{\max }$ and $A F B_{\max }$ correspond to the maximum amplitude and spectral slope, respectively, of the superficial cartilage zone, which was approximated as the range from the cartilage surface up to a depth of $300 \mu \mathrm{m}$. The decrease in the parameter values with increasing depth ( $A I B_{\text {slope }}$ and $\left.A F B_{\text {slope }}\right)$ as well as the 
extrapolation to the sample surface $z_{s}=0\left(A I B_{0}\right.$ and $\left.A F B_{0}\right)$ was quantified using linear regression analysis. The most suitable ranges of the fits were determined automatically. $A I B_{\text {slope }}$ and $A F B_{\text {slope }}$ are given in $\mathrm{dB} / \mathrm{mm}$ and $\mathrm{dB} / \mathrm{mm} / \mathrm{MHz}$, respectively, and account for the two-way propagation losses.

\section{4) Texture parameters}

The normal incidence regions were further analyzed with envelope statistics. Specifically, the homodyned k-distribution was used to model the signal amplitude of the envelope at tissue depths between 200 and $600 \mu \mathrm{m}$. A previously published algorithm was used to provide parameters from the backscattered envelope data [7]. The estimation routine provided $k$ and $\mu$ parameters from each data sample. The $k$ parameter is the ratio of coherent to incoherent signal energy and the $\mu$ parameter is related to the scatterer number density per resolution cell.

\section{E. Statistical analyses}

One-way Kruskal-Wallis non-parametric analyses of variance were carried out to assess differences of the backscatter parameters with respect to the cell Mankin score. Post-hoc multiple comparison Tukey tests were used to identify differences among subgroups. Correlations between ultrasonic and histological parameters were analyzed using linear regression. Pearson's correlation was used to determine pvalues and R-square values are provided as measures for the goodness of fit. All results were considered significant for $p<0.05$.

TABLE I. H-NUMBERS AND SIGNIFICANT SCORE DIFFERENCES OF THE ULTRASOUND PARAMETERS

\begin{tabular}{|c|c|c|c|}
\hline \multirow{2}{*}{$\begin{array}{c}\text { US } \\
\text { parameters }\end{array}$} & \multicolumn{3}{|c|}{ Mankin scores } \\
\cline { 2 - 4 } & \multicolumn{4}{|c|}{ Mankin I } & Mankin II & $\begin{array}{c}\text { cumulative } \\
\text { Mankin }\end{array}$ \\
\hline \multicolumn{4}{|c|}{ Spectral parameters } \\
\hline$A I B_{\max }$ & $\begin{array}{c}\mathbf{1 5 . 1 5} \\
(0,1>4)\end{array}$ & n.s. & $\begin{array}{c}\mathbf{1 6 . 4} \\
(2>6)\end{array}$ \\
\hline$A I B_{0}$ & n.s. & n.s. & n.s. \\
\hline$A I B_{\text {slope }}$ & n.s. & n.s. & n.s. \\
\hline$A F B_{\max }$ & $\begin{array}{c}\mathbf{2 0 . 0 8} \\
(0>1,2,3,4)\end{array}$ & $\begin{array}{c}\mathbf{1 4 . 4 4} \\
(0>1,2)\end{array}$ & $\begin{array}{c}\mathbf{1 9 . 9 1} \\
(0>3,4,6)\end{array}$ \\
\hline$A F B_{0}$ & $\mathbf{1 3 . 6 1}$ & $\mathbf{1 2 . 2 7}$ & $\mathbf{1 4 . 8 7}$ \\
$(0>2,3)$ & $(0>1,2)$ & $(0>3,5)$ \\
\hline$A F B_{\text {slope }}$ & n.s. & $\mathbf{9 . 6 5}$ & n.s. \\
\hline \multicolumn{5}{|c|}{ Envelope statistics parameters } \\
\hline$\mu$ & $\mathbf{1 3 . 9 9}$ & $\mathbf{1 0 . 3 6}$ & $\begin{array}{c}\mathbf{1 5 . 0} \\
(0>2,3)\end{array}$ \\
\hline$k$ & n.s. & n.s. & n.s. \\
\hline \multicolumn{4}{|c|}{}
\end{tabular}

\section{RESULTS}

Statistical evaluations were carried out for the described parameters with respect to Mankin I, Mankin II and the cumulative score (Table 1). Mankin II had no significant effect on the variance of all AIB parameters and only AIBmax was statistically significantly different with respect to Mankin I. In contrast, Mankin II had significant effects on all three AFB parameters. Except for AFBslope, these parameters were also statistically significantly different with respect to Mankin I. The highest significance levels and H-ratios of all investigated parameters with respect to all investigated Mankin scores were obtained for AFBmax. As shown in Fig. 1, this parameter exhibits highly positive values for healthy cartilage, but significantly lower values for samples that exhibit degenerative signs of either surface or matrix properties.
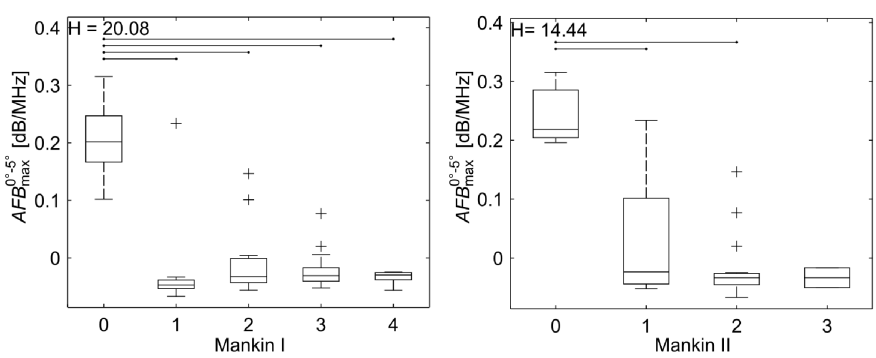

Fig. 1: Box plots of $A F B_{\max }$ with respect to Mankin I and Mankin II.

Figure 2 summarizes the relationship between cell number density and AIBmax. Linear regression revealed a positive correlation between both parameters. The cell number density with respect to Mankin II shows a slight increase from Mankin II score 0 to 1 and a decrease for higher scores (Fig. 2 b). The hyper- and hypocellularity dependent scoring criteria lead to a statistically significant difference between Mankin II grades 1 and 3. A similar trend, but with no statistically significant differences, can be observed for AIBmax (Fig. 2 c).
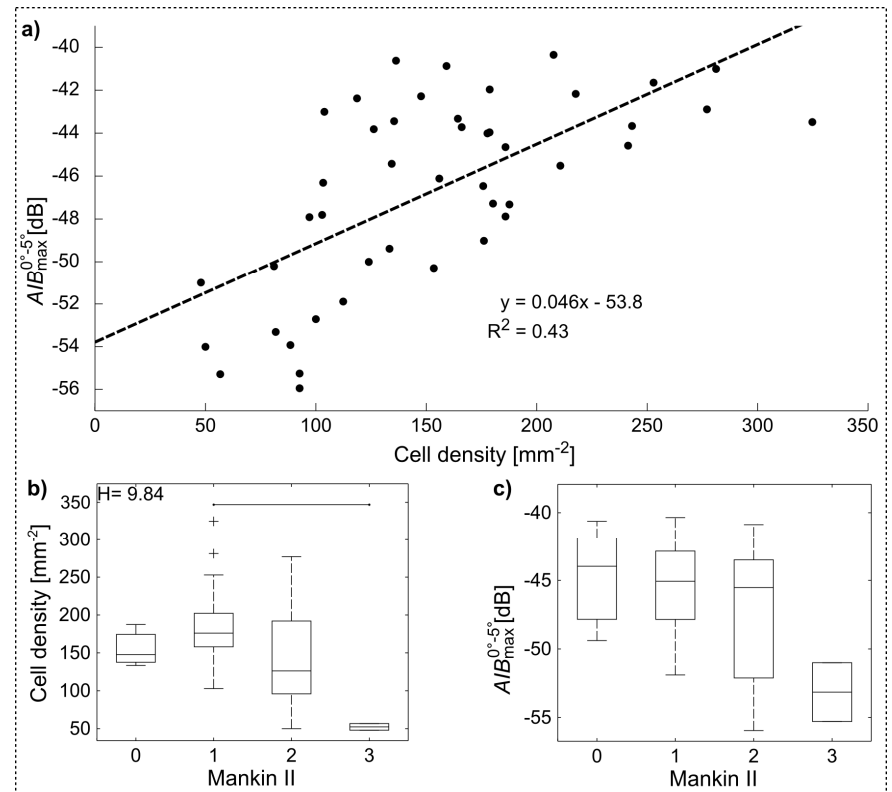

Fig. 2: Scatter plot of cell density versus $A I B_{\max }$ in conjunction with a linear regression curve (a). The corresponding coefficients as well as the goodness of fit are included. Box plots of the cell density and $A I B_{\max }$ with respect to Mankin II are shown in b) and c).

Statistically significant differences were also observed for the envelope statistics parameter $\mu$ with respect to Mankin II. In contrast to the cell number densities assessed by histology, a significant difference was observed between Mankin II grades 0 and 2 , corresponding to healthy tissue state and chondrocyte 
clustering, respectively. No significant differences were observed for $\mathrm{k}$.

\section{DISCUSSION AND CONCLUSION}

In the present study, high-frequency ultrasound backscatter signals originating from the matrix of human articular cartilage were investigated and related to spontaneous degenerative changes in the course of osteoarthritis. For the first time, in addition to amplitude parameters, spectral parameters and envelope statistics were incorporated and strictly separated in order to analyze their diagnostic value. Our results suggest that spectral and envelope statistics parameters are sensitive to osteoarthritic changes of the cartilage matrix and outperform purely amplitude-based parameters.

The region of interest was restricted to almost planar areas, which enables a link between depth dependent acoustic parameters and an idealized cross-sectional representation of collagen and chondrocytes within the different cartilage layers. It can be assumed that close to the surface, acoustic waves pass through a small tissue layer, in which the collagen fibers are aligned perpendicularly to the surface and the chondrocytes are minimal in cross section. In healthy cartilage samples, this zone showed highly positive spectral slope values and $A F B_{\max }$ exhibited the most significant variation with respect to cartilage matrix degeneration. According to scattering theory, a large positive slope of received backscatter spectra can be attributed to structures with a dimension much smaller than the wavelength $(k a<<1)$. Such structures could be both, the superficial collagen network or the high number of small chondrocytes in this tissue layer. One of the earliest signs of $\mathrm{OA}$ is the disruption of superficial collage, which could explain the diminishment of this peak. On the other hand, the moderate correlation between cell number and scattering amplitude suggests that chondrocytes are an important scattering source. Therefore, future studies should focus on the elucidation of the relative contributions of acoustic backscatter from cells and collagen matrix, as it could serve as an important biomarker for early degenerative signs at the cellular level that are not associated with a destruction of the collagen network.

The envelope statistics revealed significantly lower $\mu$ values for Mankin II grade 2compared to the healthy controls. In contrast, the cell number density assessed by histology exhibited differences between grades 1 and 3. This discrepancy results from the fact that cells have been counted individually (regardless of cell clustering) by the histology analysis, while the envelope statistics are not expected to be able to distinguish between an individual cell and a chondron containing two or more cells. Therefore, the combination of several time-domain envelope and spectral parameters may enable the identification of chondrocyte clustering.
Statistically significant differences were predominantly observed between the healthy group (Mankin I and II score 0) and all groups with Mankin scores higher than 0 . The region evaluated by histology was only a sub-region of that evaluated by ultrasound. Local changes of tissue properties are likely, particularly in the higher degeneration stages and therefore, the histological scores cannot be expected to be fully comparable to the US parameters. The limited number of samples evaluated in this study presumably prevented statistically significant differences among the groups, especially with respect to Mankin II grade $3(\mathrm{~N}=2)$. Taking this into consideration, a combination of several ultrasound parameters may enable a better discrimination between early and moderate degeneration stages.

In conclusion, it has been demonstrated for the first time that envelope statistics and depth-dependent spectral slope parameters are sensitive to the early stages of extracellular matrix degeneration and outperform conventionally used amplitude-based parameters. An application to intra-articular ultrasound arthroscopies could improve the diagnostic potential of these examinations

\section{ACKNOWLEDGMENT}

This work was supported by the German Research Council (DFG grant Ra1380/6) and Deutsche Arthrose-Hilfe e.V. N.M. receives a DFG stipend through the Berlin-Brandenburg School for Regenerative Therapies GSC 203.

\section{REFERENCES}

[1] F.L. Lizzi, M. Greenebaum, E.J. Feleppa, M. Elbaum, D.J. Coleman, "Theoretical framework for spectrum analysis in ultrasonic tissue characterization," J Acoust Soc Am, 1983, pp. 1366-1373.

[2] M.L. Oelze, W.D. O'Brien, "Application of three scattering models to characterization of solid tumors in mice," Ultrason Imaging, 2006, pp. 83-96.

[3] D.H. Agemura, W.D. O'Brien, J.E. Olerud, L.E. Chun, D.E. Eyre, "Ultrasonic propagation properties of articular cartilage at $100 \mathrm{MHz}$," J Acoust Soc Am, 1990, pp. 1786-1791.

[4] M. Schöne, N. Männicke, M. Gottwald, F. Göbel, K. Raum, "3-d highfrequency ultrasound improves the estimation of surface properties in degenerated cartilage”, Ultrasound Med Biol, 2013, pp. 834-44.

[5] H.J. Mankin, H. Dorfman, L. Lippiello, A. Zarins, "Biochemical and metabolic abnormalities in articular cartilage from osteo-arthritic human hips. II. Correlation of morphology with biochemical and metabolic data," J Bone Joint Surg Am, 1971, pp. 523-537.

[6] K. Gelse, A. Olk, S. Eichhorn, B. Swoboda, M. Schöne, K. Raum, "Quantitative ultrasound biomicroscopy for the analysis of healthy and repair cartilage tissue," Eur Cell Mater, 2010, pp. 58-71

[7] D.P. Hruska, M.L. Oelze, "Improved parameter estimates based on the homodyned K distribution," IEEE Trans Ultrason Ferroelectr Freq Control, 2009, pp. 2471-2481. 